\title{
Quadratus femoris tendinitis as a cause of groin pain
}

\author{
Pieter Klinkert jr, Robert J Porte, Theo P W de Rooij, Alexander C de Vries
}

Groin pain can be very disabling and is often difficult to diagnose. There are many possible causes for chronic groin pain. ${ }^{1-5}$ In this case report we describe a patient with quadratus femoris tendinitis, causing disabling groin pain.

\section{Case report}

A 30 year old woman, with a history of back pain, presented with pain in her right groin. The pain was not in one spot, but in a vague area in the groin. The pain first appeared six weeks previously while lifting a box of five kilograms off the ground, with her legs besides the box. This lift entails a combined movement of extension and adduction of the hip. At work she often lifts boxes of five to 10 kilograms. For exercise she jogs and works out. She recently started a leg work out, including adduction and lateral rotation exercises. She was unable to work, because of the groin pain.

Physical examination of the right groin and hip showed no signs of herniations or other abnormalities. There were no specific tender spots. The adductor stress test seemed to provoke the symptoms. An $x$ ray of the pelvis and lower spine and computer tomography of the lower spine showed no abnormalities. Initially the pain was diagnosed as adductor tendinitis. There was no improvement with the six weeks of rest and treatment with diclofenac by mouth. She then had local infiltration of the adductor tendons with Depomedrol, a combination of a longacting corticosteroid (methylprednisolone) and local anaesthetic (lidocaine-

Departments of Surgery P Klinkert jr R J Porte A C de Vries and Radiology T P w de Rooij

Westeinde Hospital, The Hague, the Netherlands

Correspondence to: Dr P Klinkert, Department of Surgery, Westeinde Hospital, PO Box 432, 2501 CK The Hague, the Netherlands.

Accepted for publication 14 May 1997

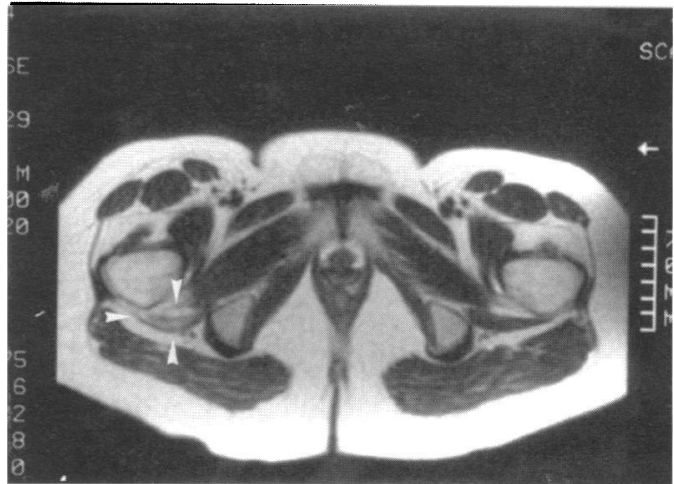

Figure 1 Axial TSE/T2 weighted magnetic resonance image showing increased signal intensity at the insertion of the right quadratus femoris tendon (arrowheads).

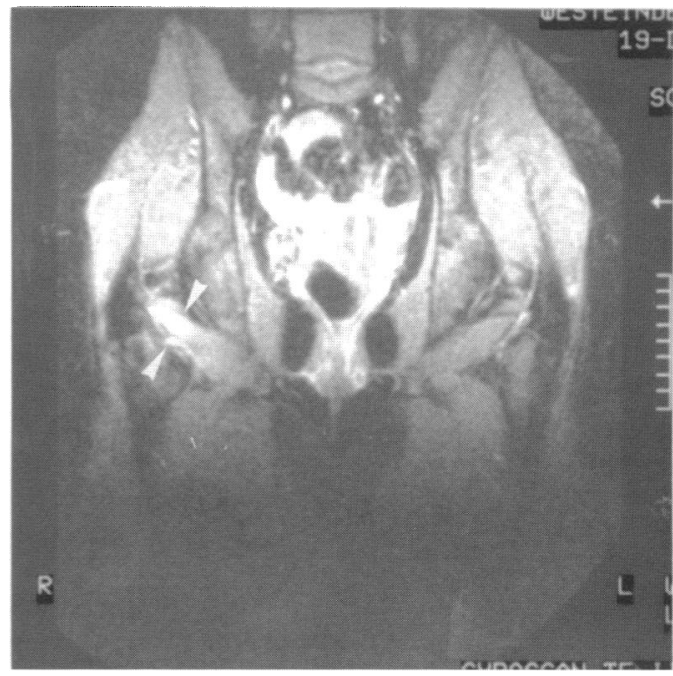

Figure 2 Coronal magnetic resonance fat suppression (STIR) image showing increased signal intensity at the insertion of the right quadratus femoris tendon (arrowheads).

work again. She has also started to jog and work out again.

\section{Conclusion}

The quadratus femoris muscle arises from the upper part of the external border of the tuberosity of the ischium and is inserted into a small tubercle of the trochanteric crest of the femur. The quadratus femoris is a lateral rotator of the thigh and helps with adduction. We think that tendinitis was caused by the recent leg work out and provoked by the lifting movement. The ment she had a gradual but complete resolution of all symptoms. The patient has now been pain free for four months and has started to 
reason for the pain in the groin may be that the tendinitis was in the trochanteric region, but that the quadratus femoris originates from the tuberosity of the ischium. It could be a referred pain.

To the authors' knowledge quadratus femoris tendinitis as a cause of groin pain has not been reported in the medical literature before. There are several publications on groin pain, especially affecting athletes. ${ }^{1-5}$ Inguinal hernia (sports hernia), tendinitis (adductor tendons, rectus femoris tendon, rectus abdominis tendon, iliopsoas tendon), bursitis, osteitis, symphysitis, and prostatitis have been described as causes of groin pain.

From this case we concluded that when physical examination and herniography do not point to the "ordinary" causes of groin pain, quadratus femoris tendinitis should be considered. Magnetic resonance imaging can confirm the diagnosis and may avoid delay in the appropiate treatment, which may be conservative with ultrasound guided local corticosteroids injections.

1 Ashby EC. Chronic obscure pain is commonly caused by enthesopathy: "tennis elbow" of the groin. $\mathrm{Br} \mathcal{F}$ Surg 1994;81:1632-4.

2 Ekberg O, Persson NH, Abrahanson P-A, Westlin NE, Lilja B. Longstanding groin pain in athletes. Sports Med 1988;6: $56-61$.

3 Karlsson J, Swärd L, Kälebo P, Thomée R. Chronic groin injuries in athletes. Sports Med 1994;17:141-8.

4 Renström PAFH. Tendon and muscular injuries in the groin area. Clin Sports Med 1992;11:815-31.

5 Zimmerman G. Groin pain in athletes. Aust Fam Physician 1988;17:1046-52.

\title{
Local complications of self administered anabolic steroid injections
}

\author{
Nick A Evans
}

\begin{abstract}
In addition to the pharmacological side effects of anabolic steroids, complications may also result from the injection technique used in self administration. Two cases are presented where anabolic steroid injections resulted in knee joint sepsis and radial nerve palsy.

(Br f Sports Med 1997;31:349-350)
\end{abstract}

Keywords: anabolic steroids; injections; complications

Intramuscular injection is a popular route of self administering anabolic steroids to improve athletic performance or improve body image. Ninety six per cent of steroid users in a recent study admitted to using injectable preparations. ${ }^{1}$ This report highlights two unusual clinical complications resulting from anabolic steroid injections, where the athletes initially chose to withhold the relevant illicit drug history.

\section{Case reports}

CASE 1

Department of

Trauma and

Orthopaedics,

Morriston Hospital,

Swansea SA9 6NL,

United Kingdom

N A Evans

Correspondence to Mr N A Evans, Department of Orthopaedics, East Glamorgan Hospital, Pontypridd CF38 1AB.
On further questioning, the athlete admitted to self administering intramuscular injections of anabolic steroid (stanozolol $50 \mathrm{mg} / \mathrm{ml}$, Zam- bon, Spain) directly into the vastus medialis in an attempt to specifically cause an increase in its size. Three separate injections of $1 \mathrm{ml}$ were administered into this area during the previous week, and although sterile needles were used, he did not seem to observe aseptic technique.

The knee joint was lavaged arthroscopically, and a small area of synovial inflammation was noted in the medial aspect of the suprapatellar pouch, presumably the site of misplaced intramuscular injections. On culture the knee joint fluid grew Staphylococcus aureus. The patient did not have any predisposition to staphylococcal infection, and made an uneventful recovery with supplemental antibiotics.

\section{CASE 2}

A 28 year old recreational bodybuilder presented to his general practitioner with a short history of parasthesia over the dorsum of his left hand. A small area of changed sensation was identified together with mild weakness (MRC grade 4) of left wrist extension. The patient linked the symptoms to a left sided neck sprain sustained while lifting weights one week earlier. With a suspicion of cervical nerve root compression, specialist opinion was sought.

When the patient was seen in clinic one month later, his neurological symptoms had resolved spontaneously. Clinical examination failed to identify any neurological deficit in the left upper limb, neck movements were full and pain free. However, skeletal muscle hypertrophy was noted, along with the traid of physical signs associated with anabolic steroid use, namely acne, gynaecomastia and striae. ${ }^{1}$ When confronted, he admitted to steroid use, and in particular, injecting nandrolone decanoate 